\title{
LES Study of Flame Stabilization in DLR Hydrogen Supersonic \\ Combustor with Strut Injection
}

\author{
Kun $\mathrm{Wu}^{1,2,3^{*}}$, Peng Zhang ${ }^{2 \dagger}$, Wei Yao ${ }^{1,3 \ddagger}$ and Xuejun Fan ${ }^{1,38}$ \\ 1. State Key Laboratory of High Temperature Gas Dynamics, Chinese Academy of Sciences, Beijing, 100190, \\ People's Republic of China \\ 2. Department of Mechanical Engineering, the Hong Kong Polytechnic University, Hong Kong \\ 3. School of Engineering Science, University of Chinese Academy of Sciences, Beijing 100049, China
}

Keywords: Scramjet; DLR; LES; Flame stabilization; Detailed reaction mechanism; hydrogen combustion

\begin{abstract}
Supersonic combustion in the hydrogen fueled DLR model scramjet combustor was computationally investigated by using Large Eddy Simulation (LES) combined with the latest detailed reaction mechanism for hydrogen combustion. Two computational models were employed including a two-dimensional reduced model and a three-dimensional model with periodicity in the spanwise direction. The two-dimensional model was fully validated against the three-dimensional model and the experimental data for the wall pressure measurements and the axial velocity under non-reacting flow condition. For reacting flow, the present model shows good agreement with the experimental axial velocity and static temperature measurements. Furthermore, radical evolution and heat release analysis were conducted both qualitatively and quantitatively to reveal the flame stabilization mechanism in the DLR combustor. The results show that the combustion is characterized by a three-stage combustion mode, namely induction, radical transportation and intense turbulent combustion stages.
\end{abstract}

\section{Nomenclature}

$H=$ height of the combustor in the isolator section

$L \quad=$ length of the whole combustor

$x=$ coordinate in the streamwise direction

$y=$ coordinate in the transverse direction

$p \quad=$ pressure

$\mathrm{T}=$ temperature

$Y_{\mathrm{i}} \quad=$ mass fraction of species $\mathrm{i}$

\footnotetext{
Ph.D student, wukun@imech.ac.cn

Assistant Professor, senior member of AIAA, pengzhang.zhang@polyu.edu.hk (Corresponding author)

Associate Professor, weiyao@imech.ac.cn

$\S$ Professor, xfan@imech.ac.cn
} 


$$
\begin{array}{ll}
d t & =\text { time step } \\
t_{f} & =\text { flow through time } \\
C_{p} & =\text { constant pressure species heat } \\
q_{c} & =\text { combustion heat of gaseous hydrogen } \\
\lambda & =\text { mixture reactivity }
\end{array}
$$

\section{Introduction}

Due to its promising performance at flight Mach numbers higher than seven, the supersonic ramjet (i.e. scramjet) fueled with hydrogen has been extensively studied in the past decades ${ }^{[1]}$. Whereas it is technically challenging and financially demanding to reproduce realistic flight conditions in ground test facilities, computational fluid dynamics (CFD) has been widely used as an alternative tool in scramjet design and analysis ${ }^{[2,3]}$. The Institute of Chemical Propulsion of the German Aerospace Center carried out various advanced measurements on hydrogen supersonic model combustor (referred to as DLR combustor hereinafter) with strut fuel injection ${ }^{[4]}$. Several advantages of the DLR combustor make it particularly suitable for validating CFD results. First, compared with other existing supersonic combustors, the DLR combustor is a small-scale laboratory model combustor and requires significantly smaller number of grids for numerical discretization, especially for large eddy simulations. Second, the DLR combustor is fueled with hydrogen and therefore substantially reduces the computational load and uncertainty of dealing with the complex chemical reaction mechanisms of hydrocarbon fuels. Finally, the systematic and comprehensive measurements were conducted to the DLR combustor under both cold and reacting flow conditions.

By virtue of the well documented experiment data, a number of numerical efforts have been devoted to study the DLR hydrogen combustor by using either Reynolds Averaged Navier-Stokes (RANS) or large eddy simulation (LES) approach. A two-dimensional RANS simulation implemented with a stretched laminar flamelet model was carried out by Oevermann ${ }^{[5]}$ to validate his numerical algorithm for turbulent diffusion flames. Regardless of the agreement between his simulation and the experimental data, the static temperature profiles in the far downstream of the strut overshot the experimental data, possibly because the overall equivalence ratio used in the simulation is higher than the experimental value. The 3D RANS and RANS/LES studies of Potturi et $a l^{[6]}$. show that the RANS approach yields better prediction to the velocity profiles than the LES/RANS approach while it predicts higher average static temperature than the latter. Berglund and Fureby ${ }^{[2]}$ conducted a LES study to identify and resolve the flow characteristics in the experiments.

In spite of these worthy numerical studies, which were mainly focused on validating numerical algorithms and combustion models, the understanding of the flame stabilization mechanism in the DLR combustor is still far from being satisfactory and the role of chemical reaction mechanism in accurately predicting the flame characteristics has not been sufficiently clarified from the viewpoint of chemical kinetics. Especially, the experiment shows that the attached DLR flame extends from the relatively low-speed recirculation zone in the immediately downstream of the strut to the farther downstream where the local flow velocities are substantially high. The deficiency of the reduced mechanisms in predicting this flame feature implies that the DLR flame 
cannot be described by using either the thermal theory or simple flame chemistry. Instead, the flame in both low- and high-speed region must be controlled by different elementary reactions and species transport in complex flow field.

Based on the above considerations, we formulated the present study aiming at exploring the flame stabilization mechanism via analyzing the species evolution and heat release rate in the DLR combustor by using the LES method with PaSR combustion model of Karlsson ${ }^{[7]}$ and the start-of-the art hydrogen combustion mechanism recently proposed by Burke et $a l^{[8]}$.. This mechanism consists 9 species and 19 reactions and has been validated against a large number of experimental data on various flames over wide ranges of temperature and pressure.

\section{Computational Specifications}

\section{A. Numerical Methodology}

The three-dimensional, filtered Navier-Stockes equations for a mixture of thermally perfect gases are numerically solved on multi-block structured meshes using finite volume method. An in-house code AstroFoam, which was developed based on the OpenFoam V 3.0 platform, was used in the present study. AstroFoam has been used in previous studies for simulating multicomponent supersonic flow, particularly for capturing shock waves and resolving turbulent eddies with high resolution ${ }^{[9,10]}$. AstroForm adopts the Kurganov and Tadmor (KT) scheme ${ }^{[11]}$ which is a second-order semi-discrete non-staggered, and central-upwind scheme. The KT scheme has been demonstrated to be able to capture the flow discontinuities (e.g. shock waves) with the non-oscillatory and low-dissipation features in the OpenFoam framework ${ }^{[12]}$. The reconstruction of all convective fluxes at faces (fluxes limiter-based) using a second-order TVD type scheme is expected to have nominal second-order accuracy in spatial integration. The adopted semi-implicit Crank-Nicholson scheme is of second order accuracy in time integration. The filtered sub-grid terms related to turbulent modeling are treated with the sub-grid scale turbulent kinetic energy one-equation model ${ }^{[13]}$ which is integrated in OpenFoam in its standard form. To model the turbulence/chemistry interaction, the present study adopts a finite-rate PaSR model by Karlsson ${ }^{[7]}$. In the present study, the recently proposed detailed hydrogen mechanism of Burke et al. ${ }^{[8]}$ was used and compared with previously used mechanisms.

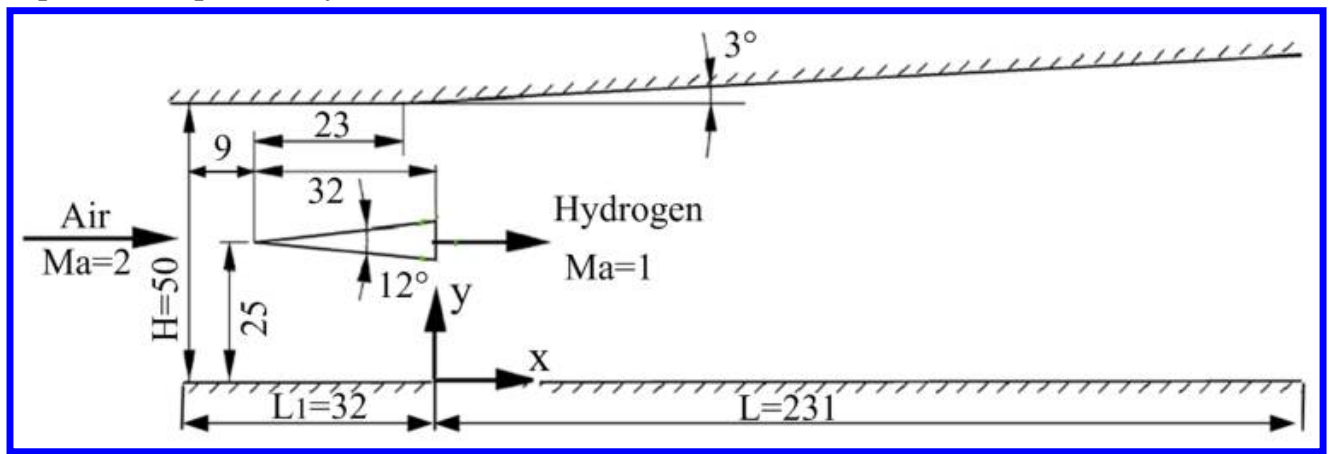

Figure 1. Schematic of the computational model of the DLR combustor (unit in mm) 


\section{B. Computational Models}

The schematic of the DLR scramjet combustor with dimensions and coordinate system is shown in Figure 1. The experiment conditions are shown in Table 1. Air is accelerated to Mach 2 through the nozzle and then enters the combustor entrance, which has a cross section of $50 \mathrm{~mm}$ in height and $40 \mathrm{~mm}$ in width. The upper wall is slightly divergent from $\mathrm{x}=-9 \mathrm{~mm}$ for three degrees to compensate for the boundary layer growth. A wedge-shape strut, which is $32 \mathrm{~mm}$ in length and has a half divergent angle of six degrees, is installed along the combustor centerline with its base located at $\mathrm{x}=0 \mathrm{~mm}$. Hydrogen is injected through an array of fifteen evenly spaced fuel orifices under sonic condition in the base of the strut. The diameter of the fuel orifice is $1 \mathrm{~mm}$ and the distance between two adjacent orifices is $2.4 \mathrm{~mm}$.

Table 1 Simulation Parameters

\begin{tabular}{|c|c|c|}
\hline Variables & Air & $\mathbf{H}_{\mathbf{2}}$ \\
\hline $\mathrm{Ma}$ & 2.0 & 1.0 \\
\hline $\mathrm{U}(\mathrm{m} / \mathrm{s})$ & 730 & 1200 \\
\hline $\mathrm{T}(\mathrm{K})$ & 340 & 250 \\
\hline $\mathrm{P}(\mathrm{Pa})$ & 100000 & 100000 \\
\hline $\mathrm{Y}_{\mathrm{O} 2}$ & 0.232 & 0 \\
\hline $\mathrm{Y}_{\mathrm{N} 2}$ & 0.736 & 0 \\
\hline $\mathrm{Y}_{\mathrm{H} 2}$ & 0.032 & 0 \\
\hline $\mathrm{Y}_{\mathrm{H} 2}$ & 0 & 1 \\
\hline
\end{tabular}

Two computational models of the DLR combustor were used in the present study. Following the previous studies, a 3D model consists of one fuel injection hole with imposed periodicity in the span-wise direction. For comparison, we developed a 2D model, in which the width of the fuel injector is equal to the diameter of the fuel orifice; a fuel-injection function was multiplied to the boundary at the fuel injector in order to keep the overall air/fuel flow rate as the same value of the experiment while retaining the local flow structure by fixing $\mathrm{Ma}=1.0$ at the fuel injector.

In the present study, the block-structured hexahedral grid was employed; the minimum mesh spacing at all solid walls is $5 \times 10^{-6} \mathrm{~mm}$ which leads to the $\mathrm{Y}^{+}$smaller than 1.0. For the 3D model, a mesh of about 3.16 million grid cells was used. For the 2D model, three meshes of about $0.19,0.27$ and 0.52 million grid cells were used for gird independence analysis. Consequently, the medium level grid with 0.27 million cells, as a balance of computational cost and accuracy, was used in the comparison with 3D results and experimental measurements, to be elaborated in the following section.

All the flow variables at the inflow boundaries are prescribed according to the theory of characteristic line and those at the outlet boundary are extrapolated from the interior. The upper, lower and strut walls are assumed to be adiabatic non-slip boundaries. The initial condition for the cold flow simulation is simply given by the main inflow throughout the domain. The initial condition for the reacting flow simulation is given by the fully developed non-reacting flow simulation. The integration time step is approximately $\mathrm{dt} \approx 5.0 \times 10^{-9} \mathrm{~s}$ which is 
limited by a maximum Courant-Friedrich-Lewy number of 0.6.

In the present study, the flow-through time is defined as $t_{f}=L / U_{\infty}=3 \times 10^{-4} s$. The $3 \mathrm{D}$ cold flow simulation runs totally for $11 t_{f}$, where the flow needs $3 t_{f}$ to reach the statistically steady state and $8 t_{f}$ is used for collecting time-averaged data. For the $2 \mathrm{D}$ model, the total simulation time is $8 t_{f}$ and $2 t_{f}$ is needed for obtaining a statistically steady state and $6 t_{f}$ for data collection. For reacting flow simulations conducted by using the $2 \mathrm{D}$ model, a longer time of $10 t_{f}$ is needed to obtain statistically steady state flow field and collecting the time-averaged data.

\section{Experimental Validation}

\section{A. Non-reacting Flow}

In the present study, a computationally efficient 2D model was proposed for detailed analysis of the DLR combustor. A 3D simulation was also employed to validate the proposed 2D model. To describe the flow field in the combustor and facilitate the following discussion, the experimental shadow graph is shown in Figure 2(a) where the time-averaged density gradient field based on the $2 \mathrm{D}$ model is also presented for comparison.

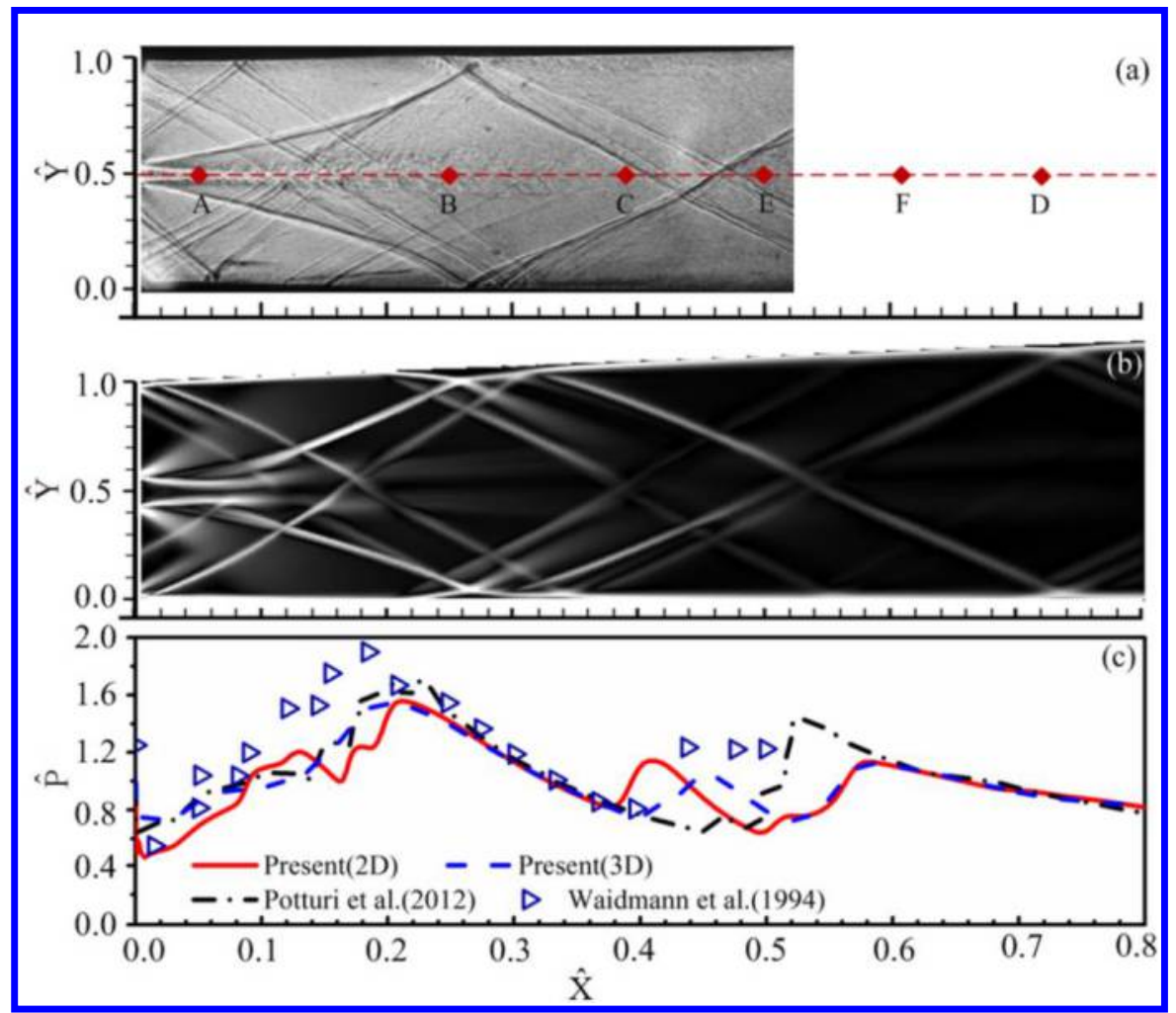

Figure 2. (a) Experimental shadow graph from Waidmann et al.(1994). (b)Time averaged density gradient contours obtained with 2D non-reacting flow model. (c)Time-averaged pressure distribution along the combustor centerline obtained using $2 \mathrm{D}$ non-reacting flow model.

It is seen that the overall shock wave patterns are well reproduced by the $2 \mathrm{D}$ simulation. To quantitatively validate the present simulations, the $3 \mathrm{D}$ and $2 \mathrm{D}$ results are compared with the experimental pressure distribution along the combustor centerline, as shown in Figure 2(c). The static pressure results are normalized using inflow air static pressure value $\mathrm{p}=10^{5} \mathrm{~Pa}$. The LES/RANS result of Potturi et al ${ }^{[14]}$. is also shown for comparison. 
Physically, the initial pressure rise shown in Figure 2(c) is due to the curving of the shear layer and the first pressure peak formed near $x / L=0.14$. The second pressure raise is induced by the impact of the reattachment of the strut leading shock waves which are captured with a reasonable agreement with the experimental result whereas under prediction in peak value. In concern with the peak value around $x / L=0.18$, the present result under predict it with $15.5 \%$ respected to the experiment measurement while the RANS/LES computation from Potturi et al ${ }^{[14]}$ gives $14.9 \%$. The 2D RANS result of Overmann ${ }^{[5]}$ presented similar trend but with even worse prediction in the peak value with $21.5 \%$ relative error while anther 2D RANS study by Hou et al ${ }^{[15]}$. performed the best to result in a $7.9 \%$ relative error. Therefore, by comparing with other computational studies relevant to DLR at the same operation condition, the present 2D LES performs reasonable well in the prediction of pressure distribution in the combustor.

\section{B. Reacting Flow}

On the fully validated non-reacting flow field, hydrogen fueled combustion phase was carried out using the detailed hydrogen oxidation mechanism of Burke et $a l^{[8]}$. Figure 3 shows the time-averaged numerical shadow graph for the reactive flow. Because of the volumetric expansion of the strut wake due to heat release, the wave pattern downstream the wedge is completely different from that of the non-reacting flow shown in Figure 2.

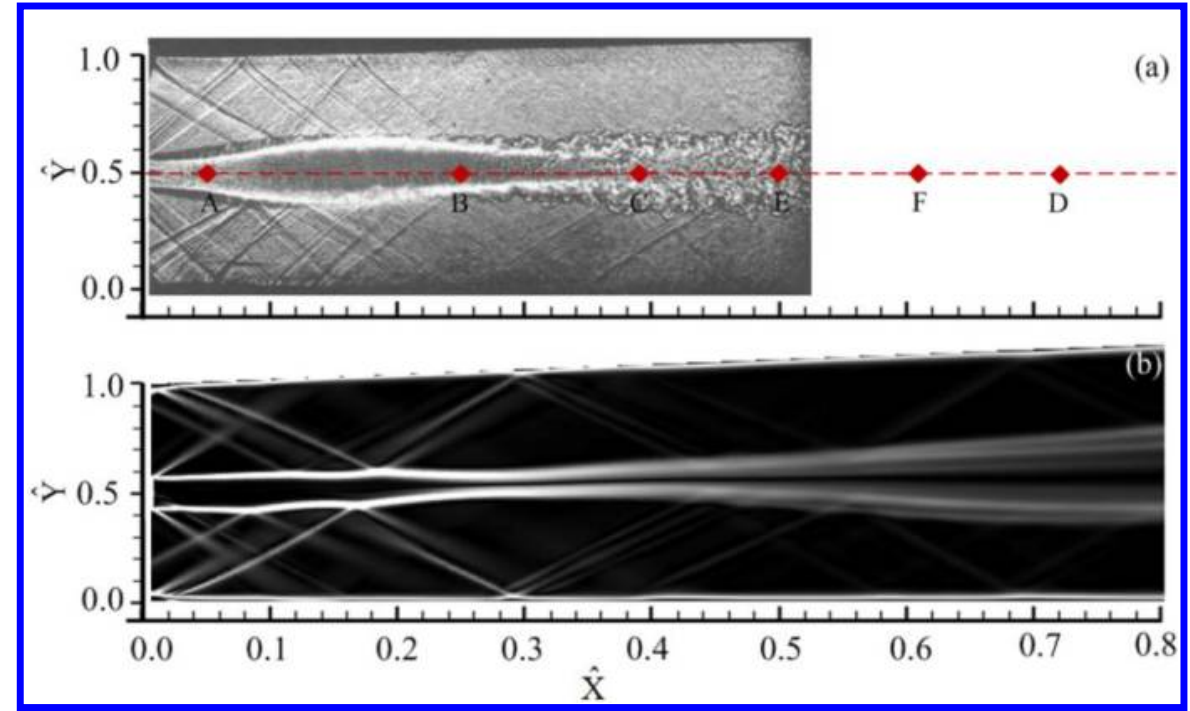

Figure 3. Time averaged density gradient contours obtained with 2D model for reacting flow compared with experimental shadow graph.

Time-averaged streamwise velocity profiles which are normalized with inflow air velocity $\left(U_{\text {air }}=\right.$ $730 \mathrm{~m} / \mathrm{s}$ ) are presented in Figure 4 and compared with experimental LDV results where available. At the location where wedge wake and fuel jet expansion dominate the velocity field, the calculated result shows big deficit in the wake flow region while velocity profiles above and below the strut are accurately captured. It seems that the numerical predicted recirculating zone in the strut wake is more intense than that of the experiment measurement resulting in a maximum reverse velocity be to $-300 \mathrm{~m} / \mathrm{s}$ while Potturi et $\mathrm{al}^{[14]}$ gave $-100 \mathrm{~m} / \mathrm{s}$. RANS study of Overmann ${ }^{[5]}$ shows similar trend but with a slighter velocity deficient in the wake region compared with the present result. 


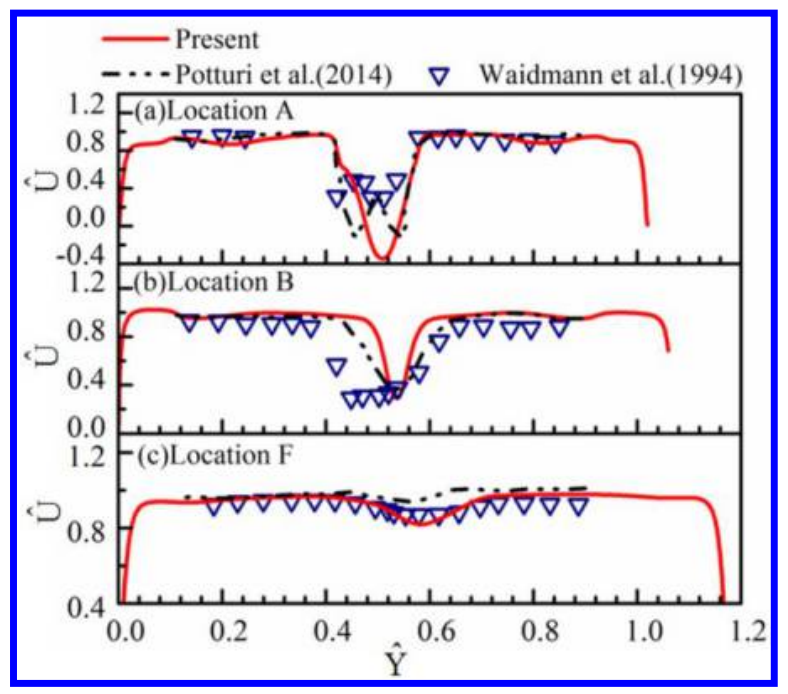

Figure 4. Time-averaged axial velocity profiles at four stream-wise locations of the reacting flow obtained using Burke's mechanism compared with LES/RANS calculation with Jachimowoski mechanism of Potturi el al.(2014)

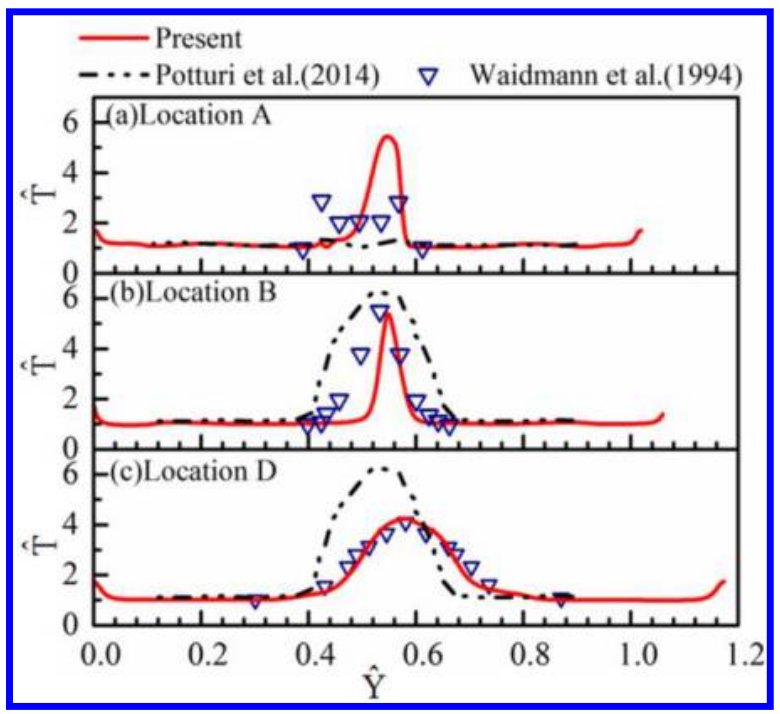

Figure 5. Time-averaged temperature profiles at four stream-wise locations of the reacting flow obtained using Burke's mechanism compared with LES/RANS calculation Potturi et al.(2014) with Jachimowoski mechanism.

The cross-section profiles of static temperature serve as an overall assessment of the reacting flow simulation with the proposed 2D model and Burke's hydrogen mechanism. In Figure 5, normalized static temperature profiles at four streamwise locations downstream the wedge strut are presented as well as CARS (Coherent Anti-Stokes Raman Spectrum) measurement. The static temperature is normalized using the combination of $c_{p} T / q_{c}$, in which $C_{p}$ and $\mathrm{q}_{\mathrm{c}}$ are constant pressure specific heat of the mixture and combustion heat of hydrogen. At the first location $\mathrm{x} / \mathrm{L}=0.048$, the temperature profile is over predicted in a very narrow region in the downstream vicinity of the strut. However, the temperature profile at the first location from Potturi et $\mathrm{al}^{[6]}$ didn't show remarkable temperature raise, which implies no ignition there and consistent with their prediction of detached flame. The good overall agreement between numerical result and experiment data at the downstream locations imply that the heat release distribution of the reacting flow is well captured by the present 
$2 \mathrm{D}$ model with detailed hydrogen oxidation mechanism of Burke et al ${ }^{[8]}$.

\section{Flame Stabilization Mechanism}

In Figure 6, time-averaged static temperature and important intermediate radicals are all presented to throw light upon the flame stabilization mechanism in the reacting flow. It can be seen from the static temperature contour that in a narrow zone in the strut wake, the local temperature reaches the highest value approximates the adiabatic flame temperature for stoichiometric hydrogen/air mixture. Further observations on Figure 6(d)-(f) reveal that there is an induction stage embedded between the fuel rich and free-stream air flow in the wake region. This is in accordance with the experimental observation that ignition occurs in the low-speed recirculation regions formed behind the wedge-shaped strut. The present result reproduced a narrower flame compared to that of Potturi et $\mathrm{al}^{[6]}$ using Jachimowoski mechanism ${ }^{[16]}$ which is also shown in the temperature profiles in Figure 5.

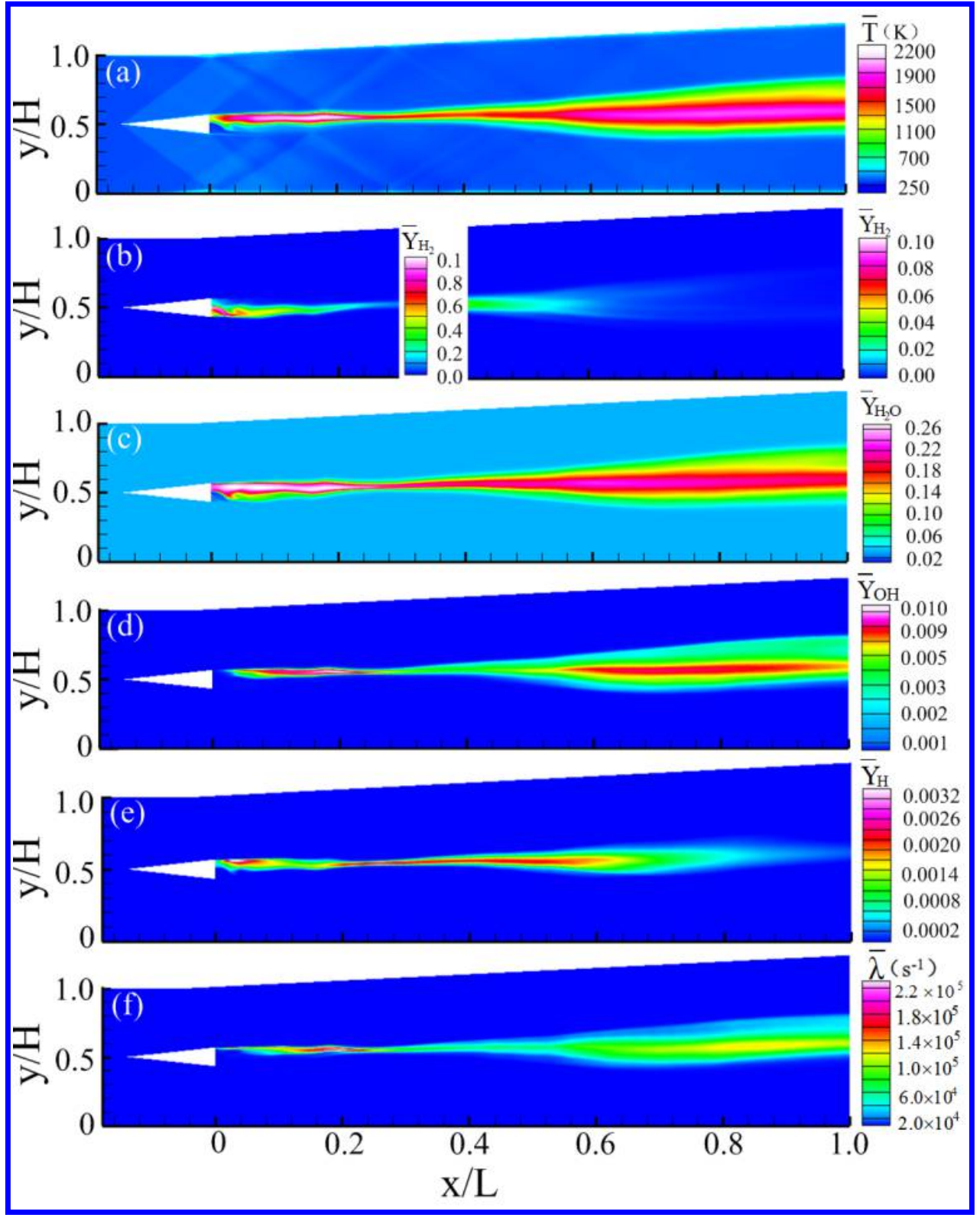

Figure 6. Contour plots of reacting flow simulation using Burke's mechanism. All variables are 


\section{time-averaged values with (a) static temperature,(b)mass fraction of $\mathrm{H}_{2}$, (c)mass fraction of $\mathrm{H}_{2} \mathrm{O}$, (d)mass fraction of $\mathrm{OH}$,(e)mass fraction of $\mathrm{H}$ and (f) mixture reactivity.}

Due to the asymmetric geometry of the combustion chamber, a fuel rich zone is formed in the lower part of the wake flow so that the reacting zone behind the wedge resides only in the upper shear layer. In the further downstream $(0.22<\mathrm{x} / \mathrm{L}<0.4)$, the free-stream flow compresses the two shear layers to a very narrow region, serving as a channel for species transportation, as shown in Figure 6(d) and (e) for $\mathrm{OH}$ and $\mathrm{H}$ radicals. In terms of the mass fraction, the $\mathrm{H}$ radical remains higher value than that in the induction stage while $\mathrm{OH}$ undergoes a reduction. After the channel region at about $\mathrm{x} / \mathrm{L}>0.4$ to the exit of the combustor, the reaction becomes intense owing to the accumulation of reactive radicals transported from the induction stage through the channel zone and then reactions becomes self-sustained due to large amount of heat release. It is confirmed by a much bigger and wider high-temperature region than the induction region in Figure 6(a). In hydrogen oxidation mechanism, the chain transfer reaction $\mathrm{OH}+\mathrm{H}_{2}=\mathrm{H}_{2} \mathrm{O}+\mathrm{H}$ is believed to be the main heat release step. As is shown in Figure 6(c) the distribution of $\mathrm{H}_{2} \mathrm{O}$ obviously distinguishes those three stages namely induction stage, transportation stage, and intense reaction stage which is in accordance with that of the static temperature shown in Figure 5(a) as it would be.

In order to illustrate the flame stabilization mechanism in this model combustor and investigate the influence of hydrogen oxidation mechanisms, the concept of mixture reactivity introduced by Bovin et $\mathrm{al}^{[17]} \mathrm{was}$ adopted to facilitate the discussion. After systematic sensitivity analysis for the three $\mathrm{H}_{2} / \mathrm{O}_{2}$ mechanisms related to auto-ignition delay, laminar flame speed, and heat release rate simulations under realistic scramjet operation condition, three most import reactions are identified. The reactivity concept manifests the competition on hydroxyl radical between the following three reactions listed in Table 2. Following Boivin et al ${ }^{[17]}$, the reactivity is defined to as follows.

$$
\lambda=2 k_{1 f} C_{O_{2}} \Lambda
$$

in which

$$
\Lambda=\left[(1+2 \mathrm{~B})^{1 / 2}-1\right] / B \text { and } \mathrm{B}=4 k_{1 f} C_{O_{2}}\left(k_{1 f} C_{O_{2}}+k_{2 f} C_{H_{2}}+k_{3 f} C_{H_{2}}\right) / k_{2 f} k_{3 f} C_{H_{2}}^{2}
$$

Table 2. H2/O2 Reaction Steps excerpt from Burke et al. 2012

\begin{tabular}{|c|c|c|c|c|c|}
\hline & Reaction & & $\mathrm{A}[\mathrm{cm}, \mathrm{g}, \mathrm{mol}, \mathrm{s}, \mathrm{K}]$ & $\mathrm{n}$ & $\mathrm{E}_{\mathrm{a}}[\mathrm{cal}, \mathrm{mol}]$ \\
\hline 1 & $\mathrm{H}+\mathrm{O}_{2}=\mathrm{O}+\mathrm{OH}$ & $k_{f}$ & $1.04 \mathrm{E}+14$ & 0.00 & $1.531 \mathrm{E}+04$ \\
\hline 2 & $\mathrm{O}+\mathrm{H}_{2}=\mathrm{H}+\mathrm{OH}$ & $k_{f}$ & $3.82 \mathrm{E}+12$ & 0.00 & $7.948 \mathrm{E}+03$ \\
\hline & Duplicate(2) & $k_{f}$ & $8.79 \mathrm{E}+14$ & 0.00 & $1.917 \mathrm{E}+04$ \\
\hline 3 & $\mathrm{H}_{2}+\mathrm{OH}=\mathrm{H}_{2} \mathrm{O}+\mathrm{H}$ & $k_{f}$ & $2.16 \mathrm{E}+08$ & 1.51 & $3.430 \mathrm{E}+03$ \\
\hline
\end{tabular}

It is obvious that mixture reactivity $\lambda$ depends only on the local reactant concentrations and temperature which gives and accurate estimation of the local non-premixed potential reactivity. By virtue of the reactivity defined above, the three-stage combustion is further confirmed. First in the strut wake, $\lambda$ begins to increase due to active $\mathrm{H}$ radical generated from chain initiation reaction $\mathrm{H}_{2}+\mathrm{O}_{2}=\mathrm{HO}_{2}+\mathrm{H}$. At location around $\mathrm{x} / \mathrm{L}=0.2, \lambda$ reaches it local maxima which indicates tendency of ignition. However, due to high flow speed in this stage, 
chemical reactions are suppressed such that this stage named as translation stage. In the third stage $(x / L=0.28)$, after accumulations of $\mathrm{OH}$ and $\mathrm{H}$ radicals, large mixture relativities occupy big portion of the combustor which characterize intense combustion. Similar flame stabilization mode was proposed by Waidmann et $\mathrm{al}^{[4]}$ in the experimental observation of DLR using spontaneous $\mathrm{OH}$ emission. They divided the flow into three stages i.e. induction stage, translational stage, and turbulent combustion stage.

\section{Concluding Remarks}

In the present work, LES was used to investigate combustion mode of the hydrogen fueled strut injection scramjet model engine. Most recent hydrogen detailed reaction mechanism combined with the finite-rate PaSR model was employed to cope the complex chemistry-turbulence interaction in the combustor. A computationally-efficient 2D model was established to facilitate a detailed study on the effects of chemical kinetics. The model was comprehensively validated against experimental data and a 3D model including one injector with periodic boundary conditions which showed the feasibility of the present 2D model. Good agreement between experimental and computational results was obtained for the wall pressure measurement, the time-averaged axial velocity, and the temperature.

By virtue of flow field and species evolution analysis, a three-stage combustion mechanism namely induction, transportation and intense turbulent combustion stage is proposed which is also accordance with experimental observation. The induction stage gives raise to the initial chemical active radials accompanied by small amount of heat release. The transportation stage serves as a channel for radical transport with very week chemical reaction. In the third stage, large amount of heat is released due to accumulation of radicals transported from upstream. This three-stage combustion mode is characterized by the radical transport and evolution which highlights the importance of the strut in the flame stabilization.

\section{Acknowledgement}

This work was supported by the Hong Kong RGC/GRF (PloyU 152651/16E) and in part by the SRFDP \& RGC ERG Joint Research Scheme (M-PolyU509/13). The Project was also supported by National Natural Science Foundation of China (Grant No. 11502270) and Training Program of the Major Research Plan of the National Natural Science Foundation of China (Grant No. 91641110). The authors are also grateful to National Supercomputer Centre in Tianjin for providing computational resource.

\section{References}

[1] Cecere D, Ingenito A, Giacomazzi E, et al. Hydrogen/Air Supersonic Combustion for Future Hypersonic Vehicles[J]. -International Journal of Hydrogen Energy, 2011, 36(18): 11969-84.

[2] Berglund M, Fureby C. Les of Supersonic Combustion in a Scramjet Engine Model[J]. Proceedings of the Combustion Institute, 2007, 31(2): 2497-504. 
[3] Gonzalez-Juez E D, Kerstein A R, Ranjan R, et al. Advances and Challenges in Modeling High-Speed Turbulent Combustion in Propulsion Systems[J]. Progress in Energy and Combustion Science, 2017, 60: 26-67.

[4] Waidmann W, Alff F, Böhm M, et al. Supersonic Combustion of Hydrogen/Air in a Scramjet Combustion Chamber[J]. Space Technology, 1995, 15(6): 421-9.

[5] Oevermann M. Numerical Investigation of Turbulent Hydrogen Combustion in a Scramjet Using Flamelet Modeling[J]. Aerospace Science and Technology, 2000, 4(7): 463-80.

[6] Potturi A S, Edwards J R. Hybrid Large-Eddy/Reynolds-Averaged Navier-Stokes Simulations of Flow through a Model Scramjet[J]. AIAA Journal, 2014, 52(7): 1417-29.

[7] Karlsson A. Modeling Auto-Ignition, Flame Propagation and Combustion in Non-Stationary Turbulent Sprays [D]; Chalmers University of Technology, 1995.

[8] Burke M P, Chaos M, Ju Y, et al. Comprehensive H2/O2 Kinetic Model for High-Pressure Combustion[J]. International Journal of Chemical Kinetics, 2011, 44(7): 444-74.

[9] Li X, Wu K, Yao W, et al. A Comparative Study of Highly Underexpanded Nitrogen and Hydrogen Jets Using Large Eddy Simulation[J]. International Journal of Hydrogen Energy, 2016, 41(9): 5151-61.

[10] Li X, Yao W, Fan X. Large-Eddy Simulation of Time Evolution and Instability of Highly Underexpanded Sonic Jets[J].

AIAA Journal, 2016, 54(10): 3191-211.

[11] Kurganov A, Tadmor E. New High-Resolution Central Schemes for Nonlinear Conservation Laws and ConvectionDiffusion Equations[J]. Journal of Computational Physics, 2000, 160(1): 241-82.

[12] Greenshields C J, Weller H G, Gasparini L, et al. Implementation of Semi-Discrete, Non-Staggered Central Schemes in a Colocated, Polyhedral, Finite Volume Framework, for High-Speed Viscous Flows[J]. International Journal for Numerical Methods in Fluids, 2009.

[13] Yoshizawa A. Statistical Theory for Compressible Turbulent Shear Flows, with the Application to Subgrid Modeling[J]. Physics of Fluids, 1986, 29(7): 2152-64.

[14] Amarnatha P, Jack E. Les/Rans Simulation of a Supersonic Combustion Experiment[J]. 50th AIAA Aerospace Sciences Meeting including the New Horizons Forum and Aerospace Exposition, 2012.

[15] Hou L, Niu D, Ren Z. Partially Premixed Flamelet Modeling in a Hydrogen-Fueled Supersonic Combustor[J]. International Journal of Hydrogen Energy, 2014, 39(17): 9497-504.

[16] Jachimowski C J. An Analytical Study of the Hydrogen-Air Reaction Mechanism with Application to Scramjet Combustion[J]. NASA Technical Paper 2791, 1988.

[17] Boivin P, Dauptain A, Jiménez C, et al. Simulation of a Supersonic Hydrogen-Air Autoignition-Stabilized Flame -Using Reduced Chemistry[J]. Combustion and Flame, 2012, 159(4): 1779-90. 